\title{
Rare but rich pickings
}

Identifying the heritable component of psychiatric conditions is one of the biggest challenges of human genetic research. A new study published in Science reveals that rare structural variants in neurodevelopmental genes might confer an increased risk to one such disease, schizophrenia.

This work points to potential genetic culprits for schizophrenia, and also forces us to revisit the prevailing hypothesis that this condition is caused by combinations of common alleles.

The idea that psychiatric illnesses might be caused by structural DNA mutations (copy number variants, or CNVs) is not new, although historically any extensive survey has been hampered by poor resolution. Microarray-based methods make it possible to detect even small changes, and indeed these approaches have identified CNVs associated with schizophrenia, and some that confer susceptibility to another mental condition, autism.

The aim of the current study was to follow up on these clues and ask whether rare CNVs were more common in individuals with schizophrenia than in controls. Over 400 individuals were screened using microarray comparative genomic hybridization. Rare deletions and duplications (>100 kb)

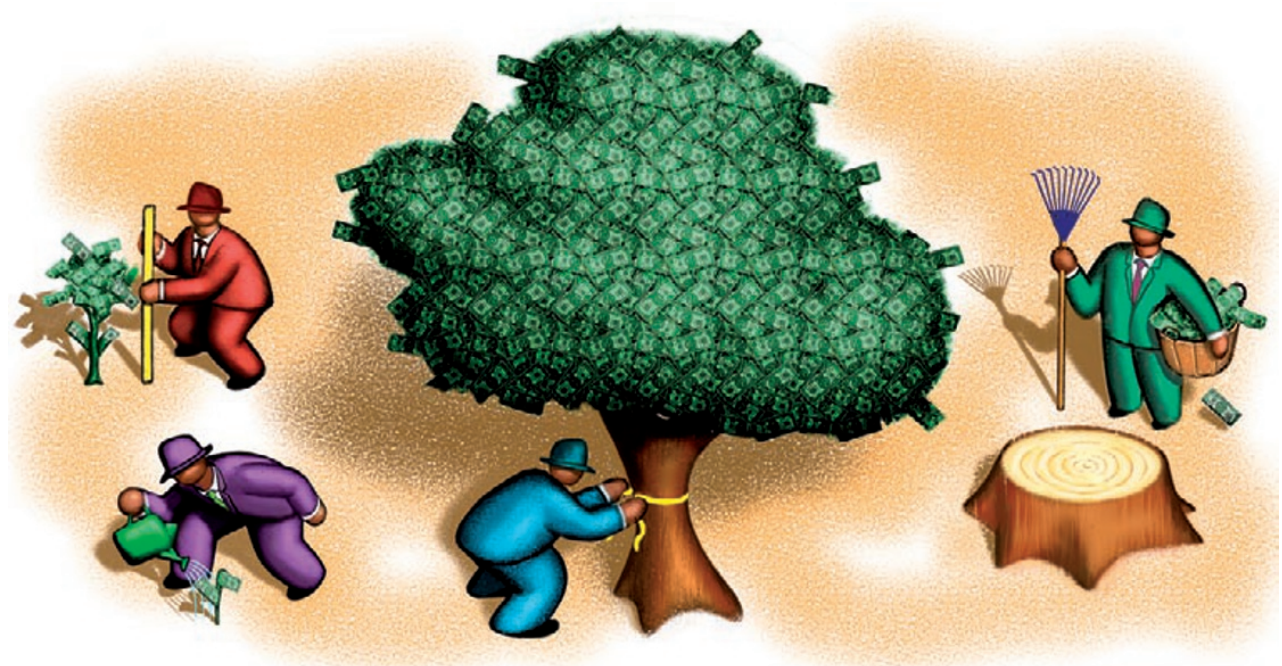

that affected genes were found in $15 \%$ of cases compared with $5 \%$ of controls. Similar results were obtained from screening a second cohort, this time consisting of 83 children suffering from childhood-onset schizophrenia.

The pattern of structural mutations is biologically revealing: CNVs in cases were more likely to disrupt genes involved in brain development, such as those that control neuronal growth and migration. Moreover, each mutation occurred in a different genomic region and involved a variety of genes.

The model suggested by this study is that many, perhaps most, cases of schizophrenia are caused by individually rare, but highly penetrant mutations - implying that most cases of schizophrenia could have a different genetic cause. A similar approach might be fruitful in the study of other psychiatric illnesses, which also might be caused by rare, large-effect mutations. 